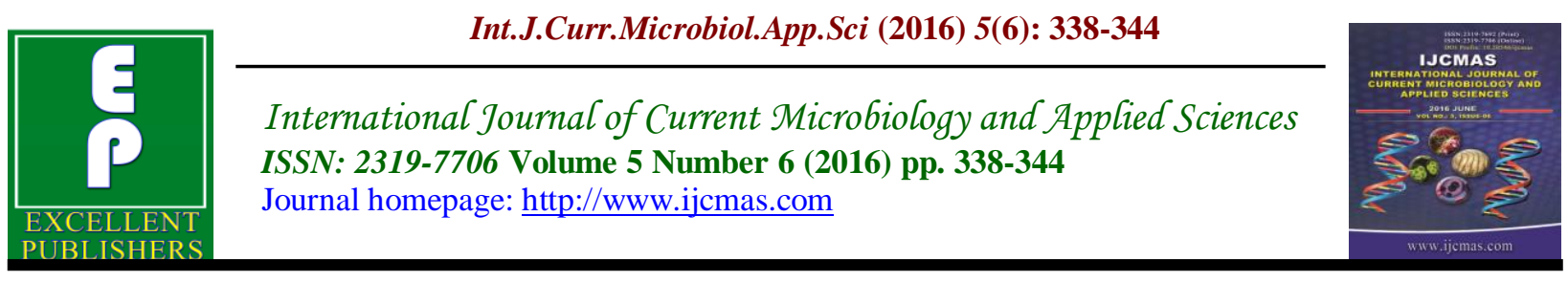

Review Article

http://dx.doi.org/10.20546/ijcmas.2016.506.038

\title{
Can a Duplex Non-treponemal and Treponemal Serological Test Help in Improving Syphilis Testing?
}

\author{
Mayur R. Shukla* \\ Department of Microbiology, Shri Jagdishprasad Jhabarmal Tibrewala University, \\ Jhunjhunu, Rajasthan-333001, India \\ *Corresponding author
}

\begin{abstract}
A B S T R A C T
Keywords

Syphilis, Treponemal test, Non-treponemal test, CLIA, ECDC.

\section{Article Info}

Accepted:

15 May 2016

Available Online:

10 June 2016

Treponemal and non-treponemal serological tests are the most commonly used for syphilis diagnosis. The traditional syphilis testing algorithm recommends the screening of samples with a non-treponemal test. Whereas, the reverse and European Centers for Disease Control (ECDC) testing algorithms advocate the use of a treponemal test for syphilis screening. The popularity of treponemal tests has recently gained interest due to their availability in the automated test format. In contrast to that, non-treponemal tests are not available in such format. Though, their usefulness cannot be underestimated. They are very helpful in determining active syphilis infection, and monitoring the progress of treatment. Hence, combing both different tests on a single platform can be very useful to those laboratories that are engaged with huge number of sample testing. Such test could possibly be designed in a duplex chemiluminescent immunoassay (CLIA) system. It can aid in improving routine syphilis screening with the advantage of fast testing, better result interpretation, and a potential for automation. In addition to that, it can satisfy the requirements of described testing algorithms. However, this duplex test needs to have sensitivity, and specificity equivalent to currently used serological tests for advancing its further use in syphilis diagnosis.
\end{abstract}

\section{Introduction}

Syphilis is a disease caused by the bacterium Treponema pallidum subspecies pallidum. It belongs to the Spirochaetaceae family and is gram-negative, helical shaped, slender, motile bacteria. This disease is transmitted majorly through sexual route. However, other modes are also possible for its transmission and includes from syphilis infected mother to her fetus while in the uterus or at the time of birth, rarely through blood transfusion, and may through nonsexual contact (Goh, 2005).
Syphilis is known for many centuries. An Italian war of 1495 (the diseases of Naples) was the reported earlier evidence. Many incidences were reported thereafter throughout the world (Frith, 2012). The origin of this disease is mysterious, and few hypotheses were proposed to understand it (Singh et al., 1999). The original Columbian hypothesis described the endemic presence of syphilis in the new world, and later was transferred to the old world through 
Columbus crew. The other hypothesis, preColumbian, stated central Africa as the place of origin for syphilis, and afterward was introduced to Europe before Columbus voyage. These two are the main theories proposed for syphilis origin. However, the Unitarian theory described that venereal and non-venereal treponematoses were derived from the single disease, and syphilis etiological agent was considered as a variant originated due to the social and environmental condition of the late $15^{\text {th }}$ century that favored its extensive transmission through sexual contact (Singh et al., 1999 and Frith, 2012). The origin of the term syphilis has an interesting history too. Earlier it was known by wide variety of names. Most were linked with the name of different countries that believed to be responsible for its spread. Later, Girolamo Fracastoro coined the term syphilis in 1530, and narrated it in his epic poem called, 'syphilis, sive morbus gallicus' (Frith, 2012).

A syphilis infection progresses through multiple stages (LaFond et al., 2006). A primary stage is characterized by the appearance of chancre, and is normally found on the genital organ. However, it can be observed on the other body sites such as oral cavity, anal canal, and rectum too. After bacterial exposure, it takes about 10 to 90 days of incubation time for its appearance (LaFond et al., 2006). During this period, bacteria invade different tissues and give rise to a symptomatic stage called secondary syphilis. The appearance of symptoms like skin rash, muscle pain, sore throat, and malaise are common during this stage. Within three months, the symptoms of secondary syphilis heal spontaneously, and are followed by an asymptomatic latent stage. This stage can be divided into an early or late latent syphilis depending upon the duration of exposure (Singh et al., 1999).
Antibiotic treatment is effective in curing this stage. However, a failure to treatment advances the infection further to the tertiary stage, which is the last stage. Gumatomatous syphilis, cardiovascular syphilis, and neurosyphilis are the outcome of the untreated infection.

Syphilis is categorized as a curable type of sexually transmitted disease due to the availability of the effective treatment options (Gottlieb et al., 2014). Penicillin is the most efficient in treating all the stages of infection. It is also useful in treating congenital syphilis. However, in case of penicillin allergy, other optional antibiotics such as ceftriaxone, erythromycin, and azithromycin are recommended (Gottlieb et al., 2014; Kingston et al., 2008; Hook et al., 2002; Riedner et al., 2005 and Blandford et al., 2003). Azithromycin is useful in treating syphilis due to its easy oral administration, and this approach is useful in resourcelimited settings (Blandford et al., 2003).

\section{Diagnosis of Syphilis}

Different techniques are used for the syphilis diagnosis. Direct microscopy from lesion, serological tests, and direct antigen detection test are the major types (Larsen et al., 1995). Among all, serological tests are the most frequently used (Ratnam, 2005). They can be grouped into non-treponemal and treponemal types (Table-1). A nontreponemal group of test deals with the detection of the non-treponemal antibody. It is produced in response to the lipid component (cardiolipin) that is thought to be derived from the damaged host cells, and from bacteria (Ratnam, 2005). It is believed that the cardiolipin is included in the outer membrane of bacteria from the host during infection (Fraser et al., 1998). The Venereal Disease Research Laboratory (VDRL) slide test, Unheated Serum Reagin (USR) test, 
Rapid Plasma Reagin (RPR) test, and Toluidine Red Unheated Serum test (TRUST) are the examples of nontreponemal tests. They all in common use an antigen suspension made up of cardiolipin, lecithin and cholesterol, and can detect $\operatorname{IgM}$ and IgG classes of non-treponemal antibodies. The presence of a nontreponemal antibody in a suspected syphilis case is an indicator of an active infection, and can aid in diagnosis (French et al., 2009). A semi-quantitative non-treponemal test can be used to determine the end-point titer of a syphilis reactive sample. A recent infection can raise the non-treponemal antibody titer, and a relapse or re-infection can give the persistent titer. A decline in antibody titer indicates recovery from the infection, and it might be due to the effectiveness of treatment (Ratnam, 2005). However, non-treponemal tests have some limitations too. A reactive sample containing large number of non-treponemal antibody can show prozone reaction. It may give a false negative result when tested undiluted (Jurado et al., 1993). Some diseases like malaria, hepatitis, viral pneumonia, chicken pox, systemic lupus erythematosus, and others can give false positive result with non-treponemal tests (Ratnam, 2005). Hence, finding obtained with a nontreponemal test needs further confirmation with a treponemal specific test (CDC, 2008). This group of test includes $T$. pallidum Hemagglutionation Assay (TPHA), $T$. pallidum Particle Agglutination Assay (TPPA), Fluorescent Treponemal Antibody Absorption assay (FTA-ABS), and treponemal antibody specific Enzyme Immunoassay (EIA) tests. These treponemal-specific tests are very sensitive and demonstrated their usefulness in diagnosing early, late or latent stage of syphilis infection (Loeffelholz et al., 2011 and Mishra et al., 2011). However, screening by a treponemal test can give false positive results in successfully treated cases (Ratnam, 2005). It might be due to the long presence of treponemal specific antibody. Hence, a result obtained with the treponemal specific test needs further confirmation with a non-treponemal test (Seňa et al., 2010).

\section{Dilemma on Syphilis Testing}

Serodiagnosis of syphilis is dependent on the acceptance of testing algorithm. The traditional algorithm recommends screening by a non-treponemal test. If the sample shows a positive result, then it is confirmed with a treponemal test (CDC, 2008). This practice has been in effect since many years and seems to be very useful so far. The current reverse algorithm is the opposite of this practice. It prefers a treponemal test for screening, and a non-treponemal test is used later for confirmation (Seňa et al., 2010). This practice is gaining importance due to the high sensitivity, and test availability in the automated format of the treponemal tests. Another type of screening practice reported recently called the ECDC algorithm (Tong et al., 2014). It uses a treponemal test for screening, and obtained finding is confirmed with another different treponemal test. A non-treponemal test can be used later for monitoring the effectiveness of treatment. All algorithms with their preference for screening and confirmatory test selection are summarized in Table- 2 .

Out of three testing algorithm described above, which one is better is subject of concern. To answer that, Tong et al. did a study with 24,124 serum samples with all described syphilis testing algorithms. Their study concluded the use of ECDC algorithm as a suitable practice, which begins with a treponemal test for syphilis screening. Contrary to that, Binnicker et al. showed concern on the use of treponemal test (reverse practice) for syphilis screening as it 
gave false positive results in low-syphilis prevalence populations in their study (Binnicker et al., 2011). Hence, selection of a screening test is a subject of debate. It may have the impact on syphilis diagnosis. This underscores the need for improving syphilis testing with a duplex test that can fulfill the requirements of different testing algorithms.

\section{Obstacles in Developing Automated Non- Treponemal Tests}

EIA and CLIA formats are the most suitable test model that can be automated easily. However, insolubility of non-treponemal cardiolipin antigen in aqueous solvent restricts its further with such test system. Few studies were reported with the nontreponemal EIA test development. Pedersen et al., (1987) reported an EIA development study with VDRL antigen. An ethanolic solution containing $0.03 \% \quad(\mathrm{Wt} / \mathrm{Vol})$ cardiolipin, $0.09 \%$ (Wt/Vol) cholesterol, and $0.021 \%$ (Wt/Vol) lecithin was evaporated on microplate surface. This antigen coated microplate was later used in test system. Described test was able to detect $\operatorname{IgM}$ and IgG classes of antibody to the coated VDRL antigen. Their report claimed an equal sensitivity, and specificity of VDRL EIA to the traditional non-treponemal tests. A second report was described based on urease enzyme based non-treponemal VDRL EIA. It was described to be capable of detecting IgG class of antibody (White et al., 1989).

A recent study described a new method in which cardiolipin was oxidized and subsequently conjugated to different molecules like biotin, BSA (Bovine serum albumin), KLH (Keyhole limpet hemocyanin), and others (Castro et al., 2011). Such prepared cardiolipin conjugates were described to be useful in lateral flow test development. A report by Shukla et al., (2015) showed a method for the covalent attachment of cardiolipin to the microplate directly and was studied in EIA system. The same antigen coating method can also be employed with CLIA platform. Hence, described studies open the gate for the nontreponemal test development with the possibility of automation.

Non-treponemal RPR and VDRL tests are in use for syphilis screening since long. They are easy to perform, cheap, available from different commercial sources, and useful in monitoring syphilis treatment effectiveness (Ratnam, 2005). However, they are not available in the automated format, and obtained results are read visually which can be subjective (Binnicker et al., 2011). A digital flocculation reader was reported that was described to be useful in reading RPR result (Castro et al., 2012). However, still a manual method is followed for sample testing. In contrast to that, EIA test format eliminate the test result subjectivity as a machine read the result (Pedersen et al., 1987). The testing protocol of an EIA can be followed easily on the automated testing system like DS2 (Dynex Technologies, USA), and can be handy in controlling intersite testing variability (Gorton et al., 2015). Hence, described obstacles of traditional non-treponemal tests can be overcome with EIA or CLIA test format.

\section{Role of Automated Duplex Test in Improving Syphilis Testing}

The screening of syphilis can made fast by using an automated test format. This approach is the most suitable for those laboratories with a huge workload. Treponemal tests are available in the automated format, and had already proved their high efficiency for syphilis screening (Donkers et al., 2014). Similarly, the availability of a sensitive and specific automated non-treponemal test can help in improving syphilis testing. Both groups of tests are valuable in syphilis diagnosis. 
Table.1 Serological tests for syphilis

\begin{tabular}{ll}
\hline Test types & Tests* \\
\hline Non-treponemal type & VDRL, USR, RPR, TRUST \\
Treponemal type & TP-HA, TP-PA, FTA-ABS, EIA \\
\hline \multicolumn{2}{c}{ *Refer to text for the abbreviations used in table. }
\end{tabular}

Table.2 Syphilis screening algorithms

\begin{tabular}{lll}
\hline Algorithm & Screening test & Confirmatory test \\
\hline Traditional & Non-treponemal & Treponemal \\
Reverse & Treponemal & Non-treponemal \\
*ECDC & Treponemal & Treponemal \\
\hline
\end{tabular}

*Refer to text for the abbreviations used in table.

Table.3 Possible interpretation scheme for automated duplex syphilis CLIA test

\begin{tabular}{lll}
\hline \multicolumn{2}{c}{ Duplex syphilis CLIA test } & \multirow{2}{*}{ Interpretation } \\
\hline Treponemal spot & Non-treponemal spot & True syphilis case \\
\hline Positive & Positive & True Negative case \\
Negative & Negative & Need further testing with a different \\
Positive & Negative & treponemal test $(C D C, 2011)$ \\
& & Biologically false positive case \\
Negative & Positive & \\
\hline
\end{tabular}

Therefore, the availability of an automated duplex treponemal and non-treponemal serological test can aid in improving syphilis screening practice. A test can be optimized in the CLIA test format at where microwell can be coated with non-treponemal and treponemal antigen as a separate spot. A report is available on the use of the multiplex EIA platform for the multiple analyte study (Liew et al., 2007). A similar platform can be utilized for the syphilis duplex test development. This test system can have several advantages. It can be sensitive, economical, reduce time of testing, and workload. Additionally, such test is capable of detecting both nontreponemal and treponemal antibody together. Hence, its availability in future can be very useful and may avoid dilemma on the testing algorithm selection. Such proposed test can screen, and confirm syphilis cases simultaneously. In case of discrepant test result, a different treponemal test such as TP-PA can be used for further verification (CDC, 2011). The possible scheme of result interpretation with the automated duplex syphilis CLIA test is shown in Table-3.

In India, RPR or VDRL tests is used for syphilis screening (Bala et al., 2013). Whereas, in the United Kingdom and Europe treponemal tests are recommended for the same (Seňa et al., 2010 and Naidu et al., 2012). This indicates that syphilis testing practice is not uniform globally. Factors like test cost, availability, and test sensitivity (Zhang et al., 2012) should also be taken into account while advocating the test use. Non-treponemal tests are very economical but lack the high sensitivity of treponemal tests. On the other hand, treponemal tests have the risk of getting false positive result in a population with low-prevalence of 
syphilis (Binnicker et al., 2011). The verification of the screening test result with another test is a time consuming and costly approach. Hence, for fast, economical, and efficient syphilis testing, a duplex nontreponemal and treponemal serological test could be an ideal solution. Such suggested format is possible with CLIA system, and it has potential for automation. However, more studies are required to make the proposed duplex test available for syphilis diagnosis.

\section{References}

Bala, M., Singh, V., Murlidhar, S., Ramesh, V. 2013. Assessment of reactivity of three treponemal tests in non-treponemal nonreactive cases from sexually transmitted diseases clinic, antenatal clinic, integrated counselling and testing centre, other different outdoor patient departments/indoor patients of a tertiary care centre and peripheral health clinic attendees. Indian J. Med. Microb., 31(3): 275-9.

Binnicker, M., Jespersen, D., Rollins, L. 2011. Treponema-specific tests for serodiagnosis of syphilis: comparative evaluation of seven assays. J. Clin. Microbiol., 49(4): 1313-7.

Blandford, J., Gift, T. 2003. The costeffectiveness of single-dose azithromycin for treatment of incubating syphilis. Sex Transm. Dis., 30(6): 502-8.

Castro, A., Wang, H. 2011. Modified cardiolipin and uses therefore. US Patent no. 7,888,043 B2.

Castro, A., Binks, D., Raymer, D., Kikkert, S., Jost, H., Park, M., et al. 2012. Evaluation of a digital flocculation reader for the rapid plasma regain test for the serological diagnosis of syphilis. Sex Transm. Dis., 39(3): 223-5.

Centers for Disease Control and Prevention. 2008. Syphilis testing algorithms using treponemal tests for initial screening-four laboratories, New York City, 2005-2006. MMWR, 57(32): 872-5.

Centers for Disease Control and Prevention.
2011. Discordant results from reverse sequence syphilis screening-five laboratories, United States, 2006-2011. MMWR Morb. Mortal Wkly. Rep., 60(5): 133-7.

Donkers, A., Levy, H., Letens-van-Vliet, A. 2014. Syphilis detection using the siemens ADVIA cenaur syphilis treponemal assay. Clin. Chim. Acta., 433: 84-7.

Fraser, C., Norris, S., Weinstock, G., White, O., Sutton, G., Dodson, R., et al. 1998. Complete genome sequence of Treponema pallidum, the syphilis spirochete. Sci., 281(5375): 375-88.

French, P., Gomberg, M., Janier, M., Schmidt, B., van Voorst Vader, P., Young, H., IUST. 2009. IUSTI:2008 European guidelines on the management of syphilis. Int. J. STD AIDS., 20(5): 300-9.

Frith, J. 2012. Syphilis- Its early history and treatment until penicillin, and the debate on its origin. J. Mil. Veterans Health, 20(4): 49-58.

Goh, B. 2005. Syphilis in adults. Sex Transm. Infect., 81(6): 448-52.

Gorton, R., White, P., Bagkeris, E., Cotterall, D., Desai, R., et al. 2015. Improved Standardization of the BioRad Platelia Aspergillus Galactomannan Antigen Sandwich EnzymeImmunoassay Using the DS2 (Dynex) Enzyme-Linked Immunosorbent Assay (ELISA) Processing System. J. Clin. Microbiol., 53: 2072-8.

Gottlieb, S., Low, N., Newman, L., Bolan, G., Kamb, M., Broutet, N. 2014. Toward global prevention of sexually transmitted infections (STI): the need for STI vaccines. Vaccine, 32 (14): 1527-35.

Hook, E., Martin, D., Stephens, J., Smith, B., Smith, K. 2002. A randomized comparative pilot study of azithromycin versus benzathine penicillin $G$ for treatment of early syphilis. Sex Transm. Dis., 29(8): 486-90.

Jurado, R., Campbell, J., Martin, P. 1993. Prozone phenomenon in secondary syphilis. Has its time arrived? Arch. Intern. Med., 153(21): 2496-8.

Kingston, M., French, P., Goh, B., Goold, P., 
Higgins, S., Sukthankar, A., et al. 2008. UK national guidelines on the management of syphilis. Int. J. STD AIDS, 19(11): 729-40.

LaFond, R., Lukehart, S. 2006. Biological basis for syphilis. Clin. Microbiol. Rev., 19: 2949.

Larsen, S., Steiner, B., Rudolph, A. 1995. Laboratory diagnosis and interpretation of tests for syphilis. Clin. Microbiol. Rev., 8(1): 1-21.

Liew, M., Groll, M., Thompson, J., Call, S., Moser, J., Hoopes, J., et al. 2007. Validating a custom multiplex ELISA against individual commercial immunoassays using clinical samples. Biotechniques, 42(3): 327-8.

Loeffelholz, M., Wen, T., Patel, J. 2011. Analysis of bioplex syphilis IgG quantitative results in different patient populations. Clin. Vaccine Immunol., 18(11): 2005-6.

Mishra, S., Boily, M., Ng, V., Gold, W., Okura, T., Shaw, M., et al. 2011. The laboratory impact of changing syphilis screening from the rapid-plasma reagin to a treponemal enzyme immunoassay: a casestudy from the Greater Toronto Area. Sex Transm. Dis., 38(3): 190-6.

Naidu, N., Bharucha, Z., Sonawane, V., Ahmed, I. 2012. Comparative study of treponemal and non-treponemal test for screening of blood donated at a blood center. Asian J. Transfus. Sci., 6(1): 32-5.

Pedersen, N., Orum, O., Mouritsen, S. 1987. Enzyme-linked immuno-sorbent assay for detection of antibodies to the venereal disease research laboratory (VDRL) antigen in syphilis. J. Clin. Microbiol., 25(9): 1711-6.
Ratnam, S. 2005. The laboratory diagnosis of syphilis. Can. J. Infect. Dis. Med. Microbiol., 16(1): 45-51.

Riedner, G., Rusizoka, M., Todd, J., Maboko, L., Hoelscher, M., Mmbando, D., et al. 2005 Single-dose Azithromycin versus penicillin $G$ Benzathine for the treatment of early syphilis. N. Eng. J. Med., 353(12): 1236-44.

Seňa, A., White, B., Sparling, P. 2010. Novel Treponema pallidum serologic tests: a paradigm shift in syphilis screening for the 21st century. Clin. Infect. Dis., 51(6): 700-8.

Shukla, M., Mody, H. 2015. Syphilis diagnosis using an advance concept for nontreponemal test development. Current Trends in Biotechnol. Pharmacy, 9(4): 344-347

Singh, A., Romanowski, B. 1999. Syphilis: review with emphasis on clinical, epidemiologic, and some biologic features. Clin. Microbiol. Rev., 12(2): 187-209.

Tong, M., Lin, L., Liu, L., Zhang, H., Huang, S., Chen, Y., et al. 2014. Analysis of 3 algorithms for syphilis serodiagnosis and implications for clinical management. Clin. Infect. Dis., 58(8): 1116-24.

White, T., Fuller, S. 1989. Visuwellregain, a non-treponemal enzyme-linked immunosorbent assay for the serodiagnosis of syphilis. J. Clin. Microbiol., 27(10): 2300-4.

Zhang, W., Yen-Lieberman, B., Means, C., Kreller, R., Waletzky, J., Daly, T. 2012. The impact of analytical sensitivity on screening algorithms for syphilis. Clin. Chem., 58(6): 1065-6.

\section{How to cite this article:}

Mayur R. Shukla. 2016. Can a Duplex Non-treponemal and Treponemal Serological Test Help in Improving Syphilis Testing? Int.J.Curr.Microbiol.App.Sci. 5(6): 338-344. doi: http://dx.doi.org/10.20546/ijcmas.2016.506.038 